\title{
OPEN BOOK DECOMPOSITIONS OF 3-MANIFOLDS
}

\author{
ROBERT MYERS ${ }^{1}$
}

\begin{abstract}
We prove that every closed, orientable 3-manifold has an open book decomposition with connected binding. We then give some applications of this result.
\end{abstract}

1. Introduction. A closed $n$-manifold has an open book decomposition if it can be constructed as follows: Let $F$ be a compact $(n-1)$-manifold with $\partial F \neq \varnothing$. Let $h$ be an autohomeomorphism of $F$ which is the identity on $\partial F$. Take $F \times[0,1]$ and identify $(h(x), 0)$ with $(x, 1)$ for $x \in F$ and $(y, 0)$ with $(y, t)$ for $y \in \partial F, t \in[0,1]$. For a manifold $M$ so constructed let $q: F \times[0,1]$ $\rightarrow M$ be the quotient map. $q(\partial F)$ is called the binding, the $q(F \times\{t\})$ are called the pages of the decomposition.

Alexander [1] proved that every closed orientable 3-manifold has an open book decomposition. It is implied in his paper, and has been widely assumed, that one can always find a decomposition with connected binding. We use a theorem proved independently by Hilden and Montesinos (stated in §2) to prove the following theorem, which was first announced in [13].

THEOREM 1. Every closed orientable 3-manifold has an open book decomposition with connected binding.

This result has been obtained independently, using different techniques, by F. González-Acuña [7].

We work throughout in either the PL or smooth category. Our terminology on braids is consistent with standard usage; we give the book by J. Birman [3] as a reference. For information on branched coverings we refer to R. H. Fox [5].

The author wishes to thank W. Jaco for help in the preparation of this paper for publication.

2. Branched coverings. The following result is widely known. See Alexander [1].

Proposition 1. Let $N$ be a closed 3-manifold having an open book decomposition with binding $A$. Suppose $f: M \rightarrow N$ is a finite sheeted covering space branched over a link $L$ such that $L \cap A=\varnothing$ and $L$ is transverse to the pages.

Presented to the Society, June 20, 1975; received by the editors February 15, 1977 and, in revised form, February 27, 1978.

AMS (MOS) subject classifications (1970). Primary 57A10; Secondary 55A25, 55A10, 57D30.

Key words and phrases. Open book decomposition, 3-manifold, fibered knot, branched covering space, braid, foliation.

'This research was partially supported by NSF Grant MCS 76-07291. 
Then $M$ has an open book decomposition with binding $f^{-1}(A)$.

We use the following presentation of a closed orientable 3-manifold $M$ as a branched covering of $S^{3}$ to construct an open book decomposition of $M$ with connected binding.

Proposition 2 (Hilden [8], [9]; Montesinos [11], [12]). Every closed orientable 3-manifold $M$ can be presented as a 3-fold dihedral covering space $f$ : $M \rightarrow S^{3}$ branched over a knot $k$.

Let $\delta_{3}=\left\langle x, y: x^{2}=y^{3}=(x y)^{2}=1\right\rangle$ be the symmetric group on three symbols. Given a closed orientable 3-manifold $M$, it follows from Proposition 2 that there is a knot $k$ in $S^{3}$ and a representation $\rho$ of $\pi_{1}\left(S^{3}-k\right)$ onto $\mathcal{S}_{3}$ such that $M$ is the completion of the covering space of $S^{3}-k$ corresponding to the subgroup $\rho^{-1}(\operatorname{gp}(x))$ of $\pi_{1}\left(S^{3}-k\right)$. Note that a meridian of $k$ is represented in $\mathcal{S}_{3}$ by an element of order two. If $J$ is an oriented simple closed curve in $S^{3}-k$, then $f^{-1}(J)$ is connected if and only if $\rho([J])$ has order three, where $[J]$ is the homotopy class of $J$ in $\pi_{1}\left(S^{3}-k\right)$.

According to Alexander [1] (see also Birman [3]), any knot in $S^{3}$ can be presented as the closed braid $\hat{\beta}$ corresponding to a geometric braid $\beta$ on $n$ strings, for some $n \geqslant 2$. Thus let $D$ be a disc and $Q_{n}=\left\{q_{1}, \ldots, q_{n}\right\}$ be a set of distinct points in int $D$. The element $\beta \in B_{n}$ determines an autohomeomorphism $h$ of $D-Q_{n}$ which is the identity on $\partial D$. So $h$ determines an open book decomposition of $S^{3}$ with binding the braid axis $A$.

If $\hat{\beta}$ presents the knot $k$, then this decomposition satisfies the conditions of Proposition 1 with respect to $f: M \rightarrow S^{3}$. Thus we get an open book decomposition of $M$ with binding $f^{-1}(A)$. In the next section we prove that the presentation of $k$ as a closed braid $\hat{\beta}$ may be chosen so that the homotopy class of the braid axis $A$ is represented in $\mathcal{S}_{3}$ by an element of order three. The theorem will then follow by the above remarks.

3. Proof of Theorem 1. Let $M$ be a closed orientable 3-manifold, with $k$ the knot in $S^{3}, \rho: \pi_{1}\left(S^{3}-k\right) \rightarrow \delta_{3}$ the representation, and $f: M \rightarrow S^{3}$ the branched covering given by Proposition 2. Let $\hat{\beta}$ be a closed braid presentation of $k$ with axis $A$. In the open book decomposition of $S^{3}$ given by $\beta$, orient $[0,1]$ from 1 to 0 , and orient $D$ so that the orientation on $D \times[0,1]$ is that of a right-handed screw. Give $A$ and $\hat{\beta}$ the induced orientations. We denote again by $D$ and $Q_{n}$ the images of $D \times\{0\}$ and $Q_{n} \times\{0\}$ in $S^{3}$.

Choose a basepoint $s_{0}$ on $\partial D$. Let $\alpha_{j}$ be a loop in $\partial D$, based at $s_{0}$, which encloses $q_{j}$ but does not enclose any other point of $Q_{n}$. Give $\alpha_{j}$ the orientation induced from that of $D$. Let $a_{j}=\left[\alpha_{j}\right] \in \pi_{1}\left(S^{3}-\hat{\beta}, s_{0}\right)$. The $\alpha_{j}$ may be chosen so that $[A]=a_{1} \cdots a_{n}$. The $a_{j}$ generate $\pi_{1}\left(S^{3}-\hat{\beta}, s_{0}\right)$ and are all conjugate. $\rho\left(a_{j}\right)=x y^{\varepsilon_{j}}$, where $0 \leqslant \varepsilon_{j} \leqslant 2$. Take a regular projection of $\hat{\beta}$ onto a plane perpendicular to $A$ and compute an over presentation [4] of $\pi_{1}\left(S^{3}-\hat{\beta}, s_{0}\right)$. The generators $a_{1}, \ldots, a_{n}$ are as indicated in Figure 1 . 


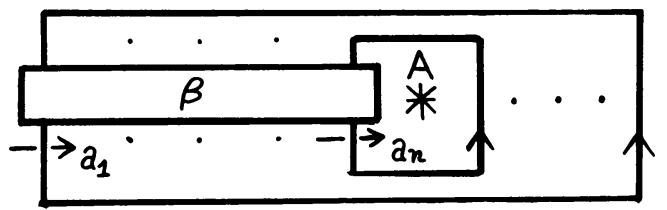

FIGURE 1

Since $\rho$ is onto and $n>1$, there is an integer $m$, with $1 \leqslant m \leqslant n-1$, such that $\rho\left(a_{m}\right) \neq \rho\left(a_{m+1}\right)$. If $n=2$, then

$$
\rho([A])=x y^{\varepsilon_{1}} x y^{\varepsilon_{2}}=y^{\varepsilon_{1}-\varepsilon_{2}} \neq 1
$$

thus $\rho([A])$ has order three and we are done. We may assume that $n$ is even, since if $n$ is odd we can replace $\beta$ by the braid $\beta \sigma_{n} \in B_{n+1}$, which also presents $k$. (The $\sigma_{i}$ are the standard Artin generators of the braid group.) So we shall now assume that $n>2$ and is even.

We may assume that $\rho\left(a_{n-1}\right) \neq \rho\left(a_{n}\right)$. If not, there is an integer $m$ as before with $\rho\left(a_{m}\right) \neq \rho\left(a_{m+1}\right)$. Let

$$
\tau_{m}=\left(\sigma_{m} \cdots \sigma_{n-1}\right)\left(\sigma_{m-1} \cdots \sigma_{n-2}\right)
$$

and $\beta^{\prime}=\tau_{m}^{-1} \beta \tau_{m}$. Let $A^{\prime}$ and $a_{j}^{\prime}$ be defined for $\hat{\beta}^{\prime}$ as $A$ and $a_{j}$ were defined for $\hat{\beta}$. A projection of $\hat{\beta}^{\prime}$ is shown in Figure 2 . Evidently there is a homeomorphism $g$ of $S^{3}$, isotopic to the identity and fixing $s_{0}$, such that $g\left(\hat{\beta}^{\prime}\right)=\hat{\beta}$ and $g_{*}\left(a_{n-1}^{\prime}\right)=a_{m-1}, g_{*}\left(a_{n}^{\prime}\right)=a_{m}$, where

$$
g_{*}: \pi_{1}\left(S^{3}-\hat{\beta}^{\prime}, s_{0}\right) \rightarrow \pi_{1}\left(S^{3}-\hat{\beta}, s_{0}\right)
$$

is induced by $g \mid\left(S^{3}-\hat{\beta}^{\prime}\right)$. Let $\rho^{\prime}=\rho \circ g_{*}$. Then $\rho^{\prime}\left(a_{n-1}^{\prime}\right) \neq \rho^{\prime}\left(a_{n}^{\prime}\right)$. So replace $\hat{\beta}$ by the equivalent knot $\hat{\beta}^{\prime}$ and drop the primes.

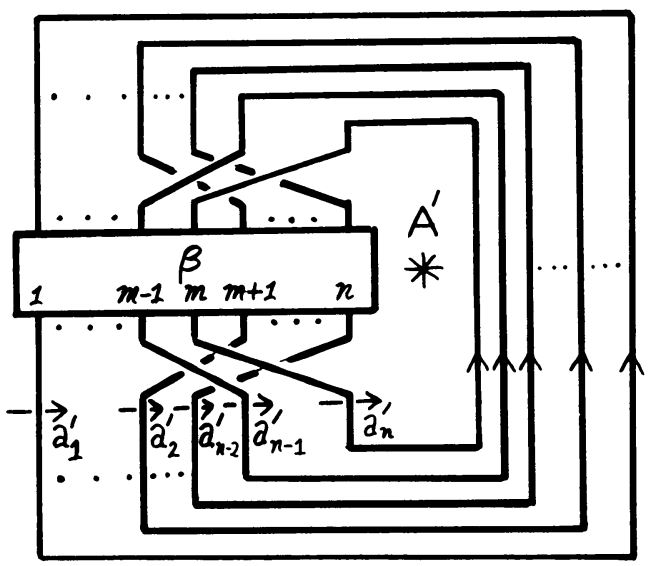

FIgURE 2

Now if $\rho([A]) \neq 1$, then it must have order three, since $n$ is even, and we are done. So assume $\rho([A])=1$. We construct a braid $\beta^{\prime} \in B_{n+2}$ such that $\hat{\beta}^{\prime}$ is equivalent to $\hat{\beta}$ and has braid axis $A^{\prime}$ with $\rho^{\prime}\left(\left[A^{\prime}\right]\right) \neq 1$, where $\rho^{\prime}$ is the 
corresponding representation of $\pi_{1}\left(S^{3}-\hat{\beta}^{\prime}, s_{0}\right)$ onto $\Im_{3}$. Let

$$
\beta^{\prime}=\beta \sigma_{n} \sigma_{n-1}^{-1} \sigma_{n}^{-1} \sigma_{n+1} \sigma_{n} \sigma_{n-1} \text {. }
$$

A projection of $\hat{\beta}^{\prime}$ is shown in Figure 3.

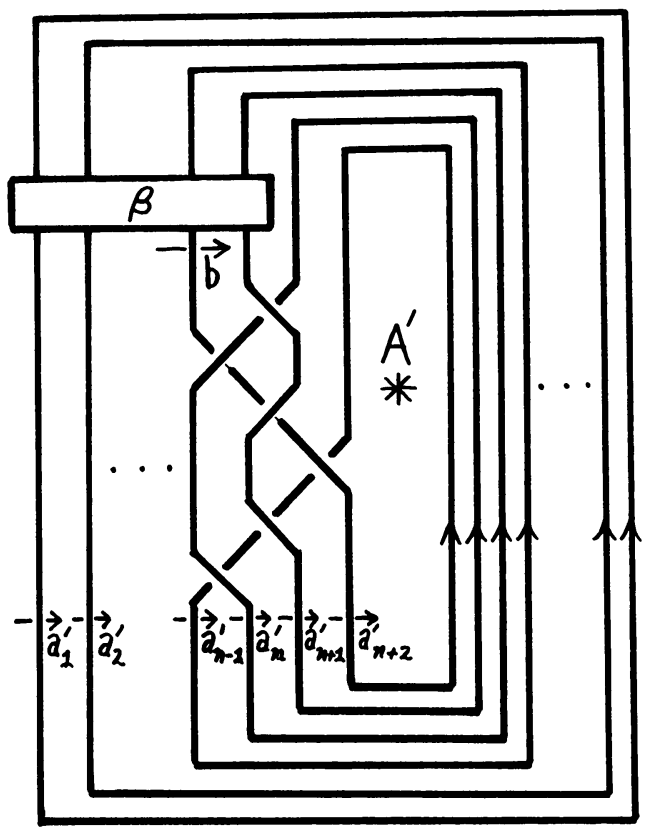

Figure 3

There is homeomorphism $g$ from $S^{3}$ to itself, isotopic to the identity and fixing $s_{0}$, such that $g\left(\hat{\beta}^{\prime}\right)=\hat{\beta}^{\prime}, g_{*}\left(a_{j}^{\prime}\right)=a_{j}$ for $1 \leqslant j \leqslant n-2, g_{*}\left(a_{n+1}^{\prime}\right)=a_{n}$, and $g_{*}(b)=a_{n-1}$, where

$$
g_{*}: \pi_{1}\left(S^{3}-\hat{\beta}^{\prime}, s_{0}\right) \rightarrow \pi_{1}\left(S^{3}-\hat{\beta}, s_{0}\right)
$$

is induced by $g \mid\left(S^{3}-\hat{\beta}^{\prime}\right)$. From the projection one calculates that

$$
\begin{aligned}
g_{*}\left(a_{n}^{\prime}\right) & =g_{*}\left(a_{n+1}^{\prime} a_{n+1}^{\prime}\left(a_{n+1}^{\prime}\right)^{-1}\right)=g_{*}\left(a_{n+1}^{\prime}\right)=a_{n}, \\
g_{*}\left(a_{n+2}^{\prime}\right) & =g_{*}\left(\left(a_{n+1}^{\prime}\right)^{-1}\left(a_{n}^{\prime}\right)^{-1} b a_{n}^{\prime} a_{n+1}^{\prime}\right)=a_{n}^{-2} a_{n-1} a_{n}^{2}, \\
g_{*}\left(a_{n-1}^{\prime}\right) & =g_{*}\left(a_{n}^{\prime} a_{n+1}^{\prime} a_{n+2}^{\prime} a_{n+2}^{\prime}\left(a_{n+2}^{\prime}\right)^{-1}\left(a_{n+1}^{\prime}\right)^{-1}\left(a_{n}^{\prime}\right)^{-1}\right) \\
& =g_{*}\left(a_{n}^{\prime} a_{n+1}^{\prime} a_{n+2}^{\prime}\left(a_{n+1}^{\prime}\right)^{-1}\left(a_{n}^{\prime}\right)^{-1}\right) \\
& =a_{n} a_{n} a_{n}^{-2} a_{n-1} a_{n}^{2} a_{n}^{-1} a_{n}^{-1}=a_{n-1} .
\end{aligned}
$$

Thus $g_{*}\left(\left[A^{\prime}\right]\right)=g_{*}\left(a_{1}^{\prime} \cdots a_{n+2}^{\prime}\right)=a_{1} \cdots a_{n-2} a_{n-1} a_{n}^{2} a_{n-1}=[A]\left(a_{n} a_{n-1}\right)$. Let $\rho^{\prime}=\rho \circ g_{*}$. Then

$$
\rho^{\prime}\left(\left[A^{\prime}\right]\right)=\rho([A]) \rho\left(a_{n}\right) \rho\left(a_{n-1}\right)=\rho\left(a_{n}\right) \rho\left(a_{n-1}\right) .
$$


Since $\left(\rho\left(a_{j}\right)\right)^{2}=1$ and $\rho\left(a_{n-1}\right) \neq \rho\left(a_{n}\right)$, it follows that $\rho^{\prime}\left(\left[A^{\prime}\right]\right) \neq 1$, and we are done.

Dropping the primes we see that $f^{-1}(A)$ is the connected binding of the open book decomposition of $M$ lifted from that of $S^{3}$ given by $\beta$. This completes the proof of Theorem 1.

\section{Applications.}

COROLlaRY 1. Every closed orientable 3-manifold contains fibered knots.

Proof. $M-f^{-1}(A)$ is a surface bundle over $S^{1}$.

The next result was first proven by $\mathrm{R}$. H. Bing [2].

COROLlaRY 2. Every closed orientable 3-manifold contains a simple closed curve whose complement is irreducible.

Proof. Surface bundles over $S^{1}$ whose fibers are not spheres are irreducible.

REMARKS. Note that the method used to prove Corollary 1 is closely related to the method used by D. Goldsmith [6] to construct fibered links in $S^{3}$. Corollary 2 shows that Theorem 1 yields an alternative proof of Bing's theorem [2] that a closed 3-manifold is $S^{3}$ if and only if each simple closed curve in $M$ is contained in a 3-cell.

COROllary 3. Every closed orientable 3-manifold has a codimension one foliation with precisely one closed leaf; this leaf is a torus.

Proof. Replace a tubular neighborhood of $f^{-1}(A)$ with a Reeb component and "turbulize" the fibers around it. (See [10] for details.)

\section{REFERENCES}

1. J. W. Alexander, A lemma on systems of knotted curves, Proc. Nat. Acad. Sci. U.S.A. 9 (1923), 93-95.

2. R. H. Bing, Necessary and sufficient conditions that a 3-manifold be $S^{3}$, Ann. of Math. 68 (1958), 17-37.

3. J. S. Birman, Braids, links, and mapping class groups, Ann. of Math. Studies, No. 82, Princeton, N.J., 1974.

4. R. H. Crowell and R. H. Fox, Introduction to knot theory, Ginn, Boston, Mass., 1963.

5. R. H. Fox, Covering spaces with singularities, Algebraic Geometry and Topology, Princeton Univ. Press, Princeton, N.J., 1957, pp. 243-257.

6. D. L. Goldsmith, Symmetric fibered links, Knots, Groups, and 3-Manifolds, Ann. of Math. Studies, No. 84, Princeton Univ. Press, Princeton, N.J., 1975, pp. 3-23.

7. F. González-Acuña, 3-dimensional open books, Lectures, Univ. of Iowa Topology Seminar, $1974 / 75$.

8. H. M. Hilden, Every closed orientable 3-manifold is a 3-fold branched covering space of $S^{3}$, Bull. Amer. Math. Soc. 80 (1974), 1243-1244.

9.

10. H. B. Lawson, Foliations, Bull. Amer. Math. Soc. 80 (1974), 369-418.

11. J. M. Montesinos, A representation of closed orientable 3-manifolds as 3-fold branched coverings of $S^{3}$, Bull. Amer. Math. Soc. 80 (1974), 845-846. 
12. _ Three-manifolds as 3-fold branched covers of $S^{3}$, Quart. J. Math. Oxford Ser. (2) 27 (1976), 85-94.

13. R. Myers, Open book decompositions of 3-manifolds, Notices Amer. Math. Soc. 22 (1975), A-651.

Department of Mathematics, Rice University, Houston, TeXas 77001

Current address: Department of Mathematics, University of Texas, Austin, Texas 78712 